Article

\title{
Multi-Objective Planning of Multi-Type Distributed Generation Considering Timing Characteristics and Environmental Benefits
}

\section{Yajing Gao *, Jianpeng Liu, Jin Yang, Haifeng Liang and Jiancheng Zhang}

School of Electrical and Electronic Engineering, North China Electric Power University, Baoding 071003, China; E-Mails: qinghemyth@yahoo.com (J.L.); j.yang8@aston.ac.uk (J.Y.); hfliang@ncepu.edu.cn (H.L.); 51350570@ncepu.edu.cn (J.Z.)

* Author to whom correspondence should be addressed; E-Mail: 51351706@ncepu.edu.cn; Tel.: +86-137-2228-2201.

External Editor: Neville R. Watson

Received: 28 July 2014; in revised form: 21 September 2014 / Accepted: 22 September 2014 / Published: 29 September 2014

\begin{abstract}
This paper presents a novel approach to multi-type distributed generation (DG) planning based on the analysis of investment and income brought by grid-connected DG. Firstly, the timing characteristics of loads and DG outputs, as well as the environmental benefits of DG are analyzed. Then, on the basis of the classification of daily load sequences, the typical daily load sequence and the typical daily output sequence of DG per unit capacity can be computed. The proposed planning model takes the location, capacity and types of DG into account as optimization variables. An improved adaptive genetic algorithm is proposed to solve the model. Case studies have been carried out on the IEEE 14-node distribution system to verify the feasibility and effectiveness of the proposed method and model.
\end{abstract}

Keywords: timing characteristics; environmental benefits; multi-type distributed generation; typical daily load sequence; typical daily output sequence

\section{Introduction}

The increasingly critical energy crisis and environmental pollution are promoting the rapid development of distributed generation (DG) technology. According to the literature, $25 \%$ to $30 \%$ of the cumulative new generating capacity around the globe before 2010 was DG [1,2]. However, a large 
amount of connected DG capacity can cause a series of impacts on the distribution networks, because of the randomness and intermittency of DG outputs [3-5]. In order to compensate for the lack of independent power generation, various types of DG are always connected to produce electricity complementally [6-8]. Therefore, multi-type DG planning is an essential problem that requires a solution by accurately considering the timing characteristics of DG outputs. Only in this way can the economic and technical indicators of distribution networks be truly reflected, to achieve an optimal allocation of distributed energy resources.

DG usually refers to independent power supplies with power ranges from several kilowatts to 50 megawatts. They are small and modular, distributed near the load and compatible with the environment $[9,10]$. Because of the advantages, such as flexibility, high-efficiency and environment friendliness, this new type of energy source has become an important supplement to the traditional power grid and may potentially replace some conventional power generation modes that are low in efficiency, yet high polluting [11]. However, the environmental values of DG have not been well embodied, because the power generation costs of most power generation companies have not taken the environmental cost into consideration. In this case, conventional coal-fired power plants have developed rapidly, which interferes with the development and utilization of clean and renewable energy [12]. Therefore, it is important for DG planning to consider environmental benefits, and only in this way can the values of DG be evaluated more objectively, so that the planning result is closer to the actual situation.

In recent years, the literature on DG planning is from the direction of considering various factors and objectives. For example, the model in [13] considered electricity purchasing cost, power shortage cost, power loss cost and the cost of delaying the upgrade of the power grid. Li et al. [14] considered the timing characteristics of loads and DG outputs and established a multi-objective optimization model, including power loss and reliability based on the analysis of many scenes. In [15], three objectives containing active loss, voltage level improvement and environment improvement were reported to determine the optimal installation locations of DG. Li et al. [16] took the emissions of pollution gases, the total cost of the distribution network and the grid voltage deviation into account as objective functions and introduced the adaptive multi-objective particle swarm optimization (AMOPSO) to solve the model. In [17], a DG allocation optimization model to minimize the operation cost of DG and the network loss and to maximize the environmental benefits of DG was presented. In both [16] and [17], the authors did not consider the timing characteristics of loads and DG outputs. In [18], the authors proposed an optimization model to minimize the net present value of some economic and environmental indicators, such as costs of line upgrade, energy losses, emissions from both the grid and DG units, and so on. The model can effectively reduce the costs and gas emissions, but it cannot quantify the economic benefits of DG. In addition, it did not consider electricity trading and subsidies, so it does not apply to different types of DG owners. Xu et al. [19] considered timing characteristics and modelled DG planning in a microgrid utilizing the investment cost of DG, fuel cost, power loss cost and environmental compensation cost as the objective function. As far as the authors are aware, the economic and environmental benefits of DG have not been comprehensively modelled. Besides, single-objective optimization mode is not conducive to focusing study on the impacts of one or several indicators on DG planning. Thus, the current modelling has limitations to some extent.

In this paper, based on the consideration of the timing characteristics of loads and DG outputs, combined with the power generation costs of traditional power plants, through a comprehensive analysis of the income items and investment items of grid-connected DG and from such aspects, as loss reduction, 
delaying the upgrade of lines, environmental protection, saving fuels, electricity trading and subsidies and the fixed investment and maintenance of DG, a multi-objective optimization model for the site selection, type selection and capacity determination of DG has been established. Finally, an improved adaptive genetic algorithm is proposed to analyze the IEEE 14-node distribution system, which verifies the feasibility and effectiveness of the proposed method and model.

\section{Timing Characteristics of Load and DG Output}

\subsection{Timing Characteristics of Load}

The timing characteristics of load are mainly related to date types and meteorological factors [20,21]. Date types could be weekdays, weekends and holidays. On weekdays and weekends, power load generally varies cyclically, but on holidays, such as New Year's Day, Chinese Spring Festival, May Day, etc., obviously, the power load always presents different changes [22,23]. Meteorological factors mainly include temperature, humidity, wind speed, solar irradiation, precipitation, etc. Their impacts on the timing characteristics of load also change with seasons. Figure 1 shows the timing characteristics of load in a certain area under different date types in spring. It can be seen that the timing characteristics of loads under different date types have high offset differences. In general, the load on weekdays is the highest, followed by that of weekends, and the load on holidays is always the lowest.

Figure 1. Timing characteristics of loads under different date types.

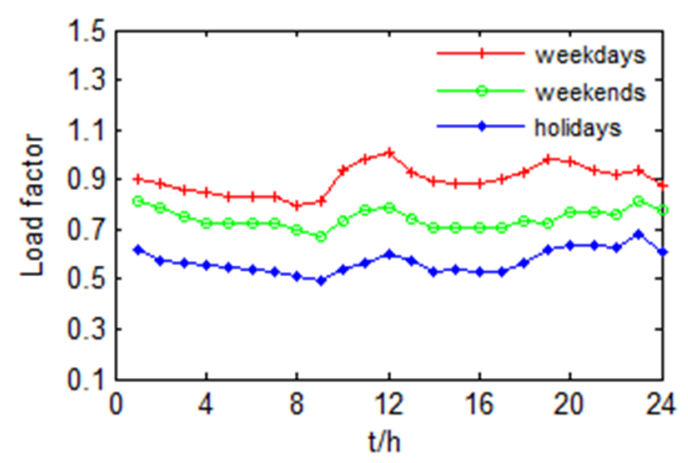

\subsection{Timing Characteristics of DG Output}

DG can be divided into two categories: renewable and non-renewable [24]: the renewable-type DG, which includes wind energy, solar energy, geothermal energy, ocean energy, etc.; and the non-renewable-type DG, like micro gas turbine, fuel cell, internal combustion engine, cogeneration, and so on.

\subsubsection{Renewable-Type DG}

For this type, wind power and photovoltaic power are the most typical, so both will be analyzed. The timing characteristics of wind power output mainly depend on the change of wind speed, where uncertainty of wind speed causes the randomness and intermittency of wind power output. According to statistics, daily variation characteristics of wind speed are mainly affected in accordance with season [25]. The timing characteristics of wind power output in different seasons are shown in Figure 2. As can be seen, wind power is lower than the rated power for most times of the day with clear randomness and volatility. 
Figure 2. Timing characteristics of wind power output in different seasons.

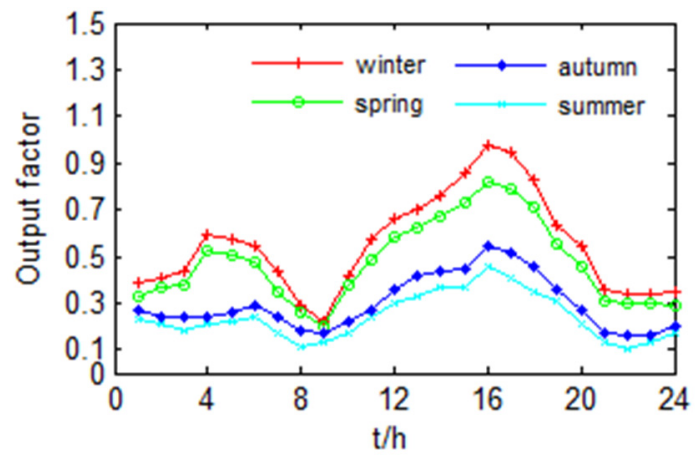

The timing characteristics of photovoltaic output are mainly affected by light intensity. In general, the daily variation characteristics of light intensity are related to season types: summer is the strongest, followed by spring and autumn, and winter is the weakest. The timing characteristics of photovoltaic output in different seasons are shown in Figure 3. It can be seen that photovoltaic output has obvious intermittency and strong regularity.

Figure 3. Timing characteristics of photovoltaic output in different seasons.

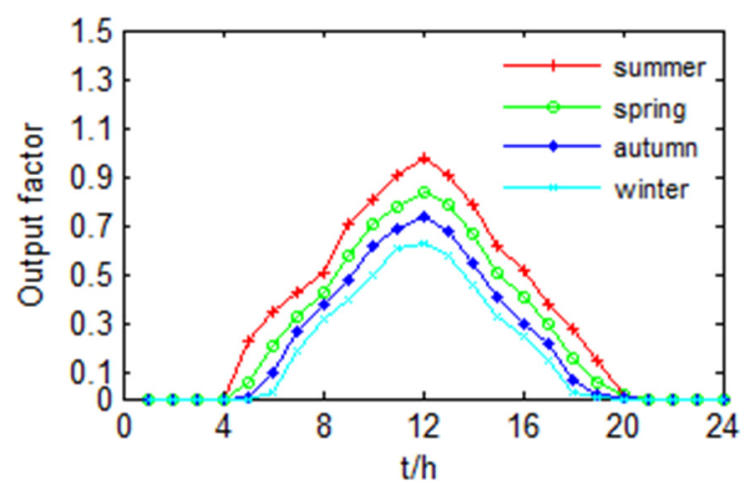

\subsubsection{Non-Renewable-Type DG}

Due to the controllability of this type, they are always used by network operators as a supplement to the former type to ensure the power balance and stability of the grid. Figure 4 shows the scheduling discipline of a wind-gas turbine hybrid power system.

Figure 4. Scheduling discipline of a wind-gas turbine hybrid power system.

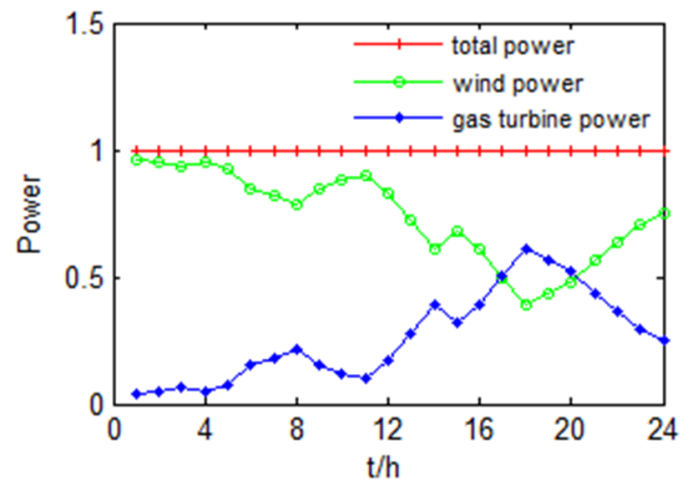




\section{Multi-Objective Planning Model of Multi-Type DG}

\subsection{Normalization of Original Data}

\subsubsection{Classification of Daily Load Sequences}

In view of the change rules of load mentioned previously, daily load sequences throughout the year can be divided into several categories according to different seasons and date types. In each category, the daily load sequences have similar change rules, which makes it possible for them to be further normalized so that the computation requirement can be reduced. The classification method of daily load sequences is shown in Figure 5.

Figure 5. Schematic diagram of the classification of daily load sequences.

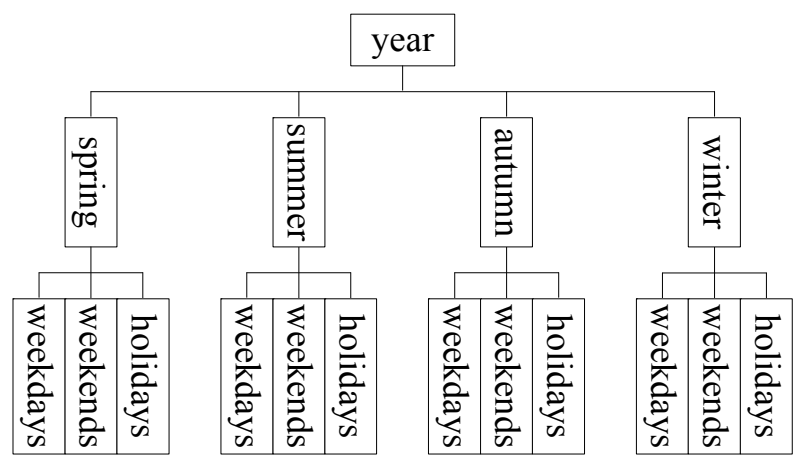

\subsubsection{Calculation of Typical Daily Load Sequence}

The typical daily load sequence can be obtained through the normalization of a certain number of daily load sequences, as in Equation (1)

$$
F_{i}=\frac{1}{n_{l}} \sum_{j=1}^{n_{l}} f_{j i}(i=1,2, \cdots, m)
$$

where $F_{i}$ is data point $i$ on the typical daily load sequence; $n_{l}$ is the total number of daily load sequences; $f_{j i}$ is data point $i$ on daily load sequence $j$; and $m$ is the total number of data points on the typical daily load sequence.

\subsubsection{Calculation of Typical Daily Output Sequence of DG per Unit Capacity}

The typical daily output sequence of DG per unit capacity can be obtained through the normalization of a certain number of daily output sequences of DG:

$$
H_{i}=\frac{1}{s n_{d}} \sum_{j=1}^{n_{d}} h_{j i}(i=1,2, \cdots, n)
$$

where $H_{i}$ is data point $i$ on the typical daily output sequence; $s$ is the capacity of the DG; $n_{d}$ is the total number of daily output sequences; $h_{j i}$ is data point $i$ on daily output sequence $j$; and $n$ is the total number of data points on the typical daily output sequence. 


\subsection{Objective Function}

The objective function is to measure and maximize the net income brought by grid-connected DG, considering the income items and investment items of DG, instead of the conventional simple minimization of indicators, like network loss, costs, etc. This is conducive in terms of not only quantifying the economic benefits, but also to focus on the impacts of one or several indicators on DG planning, so that all parties are able to make their own decisions on the planning scheme with their interested indicators. The involved income items include loss reduction, delaying the upgrade of lines, environmental protection, saving fuels, electricity trading and subsidies; and the investment item is the fixed investment and maintenance of DG.

(1) Loss reduction.

$$
C_{\text {Loss }}=c_{1} \sum_{k=1}^{N_{S}} d_{k} \sum_{i=1}^{N_{T}} t_{i} \sum_{l=1}^{N_{L}}\left(I_{k i l}^{2}-I_{k i l}^{\prime 2}\right) R_{l}
$$

In Equation (3), $C_{\text {Loss }}$ is the cumulative income of loss reduction throughout the year; where $c_{1}$ is the price of the lost electricity; $N_{S}$ is the number of typical daily load sequences; $d_{k}$ is the number of daily load sequences within typical daily load sequence $k ; N_{T}$ is the number of the time-steps divided on the typical daily load sequence; $t_{i}$ is the duration of period $i, N_{L}$ is the number of branches in the distribution network; $I_{k i l}$ and $I$ ' $k i l$ are the electric current of branch $l$ in period $i$ on typical daily load sequence $k$ before and after the connection of DG, respectively; and $R_{l}$ is the resistance of branch $l$.

(2) Delaying the upgrade of lines.

$$
C_{U p}=\sum_{i=1}^{N_{L}} e_{i}\left(\frac{r(1+r)^{n_{1 i}}}{(1+r)^{n_{1 i}}-1}-\frac{r(1+r)^{n_{2 i}}}{(1+r)^{n_{2 i}}-1}\right) C_{L i}
$$

In Equation (4), $C_{U p}$ is the income coming from delaying the upgrade of lines; where $e_{i}$ is a $0-1$ variable; $r$ is the annual interest rate; $n_{1 i}$ and $n_{2 i}$ are the number of years from the start of line $i$ to the upgrade without and with DG, respectively; and $C_{L i}$ is the fixed investment of line $i$.

(3) Environmental protection. In general, the pollutants of traditional coal-fired power plants mainly include $\mathrm{SO}_{2}, \mathrm{NO}_{x}, \mathrm{CO}_{2}, \mathrm{CO}$, total suspended particulate (TSP), fly ash, slag, etc. The development and utilization of DG can effectively reduce the emissions of these pollutants, so that the environment can be improved to a certain extent. The costs of environmental values caused by these pollutants from the production of each unit of electricity can be calculated using the data provided in [26]. Table 1 shows the calculation results of traditional coal-fired power plants and some common DG.

Table 1. Costs of environmental values of various pollutants (Chinese Yuan (CNY)/MWh).

\begin{tabular}{cccccccc}
\hline Power generation methods & $\mathbf{S O}_{2}$ & $\mathbf{N O}_{\mathbf{x}}$ & $\mathbf{C O}_{\mathbf{2}}$ & $\mathbf{C O}$ & $\mathbf{T S P}$ & Fly ash & Slag \\
\hline Coal-fired power & 41.47 & 23.04 & 27.42 & 0.09 & 0.32 & 47.52 & 1.08 \\
Wind power & 0 & 0 & 0 & 0 & 0 & 0 & 0 \\
Photovoltaic power & 0 & 0 & 0 & 0 & 0 & 0 & 0 \\
Gas turbine & 0.01 & 9.92 & 17.69 & 0 & 0.10 & 0 & 0 \\
Fuel cell & 0.01 & 7.75 & 13.82 & 0 & 0.08 & 0 & 0 \\
\hline
\end{tabular}


The environmental benefits of DG can be expressed as follows:

$$
\begin{gathered}
C_{E n v}=\sum_{a=1}^{N_{D}} Q_{a} \sum_{i=1}^{N_{P}}\left(C_{F P i}-C_{D P i}\right) \\
Q_{a}=\sum_{k=1}^{N_{S}} d_{k} \sum_{j=1}^{N_{T}} t_{j} P_{a k j}
\end{gathered}
$$

where $C_{E n v}$ is the income coming from environmental production; $N_{D}$ is the number of grid-connected DG generators; $Q_{a}$ is the annual power generation of the $a$-th DG generator; $N_{P}$ is the number of the types of pollutants; $C_{F P i}$ and $C_{D P i}$ are the cost of environmental values caused by pollutant $i$ from traditional coal-fired power plants and DG, respectively; and $P_{a k j}$ is the active power in period $j$ on typical daily load sequence $k$ of the $a$-th DG generator.

If only the interests of the DG owners are considered, pollution fines should be applied by the market regulator. In this case, the benefits of environmental values do not belong to the owner of DG. Equation (5) can be rewritten as:

$$
C_{E n v}=-\sum_{a=1}^{N_{D}} Q_{a} \sum_{i=1}^{N_{P}} D_{P i}
$$

where $D_{P i}$ is the fine of pollutant $i$.

(4) Saving fuels.

$$
C_{\text {Fuel }}=\sum_{a=1}^{N_{D}} Q_{a}\left(C_{F}-C_{D a}\right)
$$

In Equation (8), $C_{F u e l}$ is the income coming from saving fuels; where $C_{F}$ is the average cost of the fuels consumed by traditional coal-fired power plants per unit electricity produced; $C_{D a}$ is the average cost of the fuels consumed by the $a$-th DG generator when a unit of electricity is produced.

Similarly, if only the interests of the DG owners are considered, Equation (8) should be rewritten as:

$$
C_{\text {Fuel }}=-\sum_{a=1}^{N_{D}} Q_{a} C_{D a}
$$

(5) Electricity trading and subsidies. This income item exists only when only the interests of the DG owners are considered; otherwise, it does not exist.

$$
\begin{gathered}
C_{\text {Sasb }}=\left(\alpha C_{B u y}+\beta C_{S a l}+C_{S u b}\right) \sum_{a=1}^{N_{D}} Q_{a} \\
\alpha+\beta=1
\end{gathered}
$$

where $C_{\text {Sasb }}$ is the income obtained by the owner of DG coming from electricity trading and subsidies; $C_{B u y}$ is the purchase price of the electricity; $C_{S a l}$ is the sell price of the electricity; $C_{S u b}$ is the subsidy price of the electricity; and $\alpha$ and $\beta$ are, respectively, the proportion of the electric quantity to buy and that to sell.

(6) The fixed investment and maintenance of DG.

$$
C_{D G}=\sum_{i=1}^{N_{D}}\left(\frac{r(1+r)^{n_{D i}}}{(1+r)^{n_{D i}}-1} V_{D i}+W_{D i}\right)
$$


where $C_{D G}$ is the fixed investment and maintenance cost of DG converted to each year; $n_{D i}$ is the economic life of the $i$-th DG generator; $V_{D i}$ is the fixed investment cost of the $i$-th DG generator; and $W_{D i}$ is the maintenance cost of the $i$-th DG generator each year.

Based on the above analysis, the objective function in this paper can be expressed as:

$$
\max C=\lambda_{1} C_{\text {Loss }}+\lambda_{2} C_{U p}+\lambda_{3} C_{E n v}+\lambda_{4} C_{F u e l}+\lambda_{5} C_{\text {Sasb }}-\lambda_{6} C_{D G}
$$

As can be seen, when $\lambda_{1}=\lambda_{2}=\lambda_{3}=\lambda_{4}=\lambda_{6}=1$ and $\lambda_{5}=0$, Equation (13) is the maximum net income brought by DG. In addition, when different interest groups are making decisions on the planning scheme, they can adjust the corresponding weights according to their interested indicators so that their own benefits can be maximized.

\subsection{Constraint Conditions}

Constraints for the proposed planning model are listed as below:

$$
\begin{gathered}
P_{G i}+P_{D i}-P_{L i}=U_{i} \sum_{j=1}^{N} U_{j}\left(G_{i j} \cos \theta_{i j}+B_{i j} \sin \theta_{i j}\right) \\
Q_{G i}+Q_{D i}-Q_{L i}=U_{i} \sum_{j=1}^{N} U_{j}\left(G_{i j} \sin \theta_{i j}-B_{i j} \cos \theta_{i j}\right) \\
U_{i}^{\min } \leq U_{i} \leq U_{i}^{\max } \\
P_{D i}^{\min } \leq P_{D i} \leq P_{D i}^{\max } \\
P_{D}^{\min } \leq \sum_{i=1}^{n_{L}} P_{D i} \leq P_{D}^{\max } \\
P_{D k}^{\min } \leq \sum_{i=1}^{n_{L}} P_{D k i} \leq P_{D k}^{\max }
\end{gathered}
$$

where $P_{G i}, P_{D i}$ and $P_{L i}$ are, respectively, the active power of the generator, DG and load at node $i, Q_{G i}$, $Q_{D i}$ and $Q_{L i}$ are, respectively, the reactive power of the generator, DG and load at node $i, G_{i j}$ and $B_{i j}$ are, respectively, the conductance and susceptance of branch $i j, \theta_{i j}$ is the power angle between node $i$ and node $j, U_{i}, U_{i}^{\max }$ and $U_{i}^{\min }$ are, respectively, the voltage and its upper and lower limits at node $i, P_{D i}$, $P_{D i}^{\max }$ and $P_{D i}^{\min }$ are, respectively, the active power and its upper and lower limits of the DG at node $i$, $P_{D}^{\max }$ and $P_{D}^{\min }$ are, respectively, the upper and lower limits of the DG in the distribution network, $n_{L}$ is the number of load points in the distribution network, $P_{D k i}$ is the active power of the DG whose type number is $k$ at node $i$ and $P_{D k}^{\max }$ and $P_{D k}^{\min }$ are, respectively, the upper and lower limits of the active power of the DG whose type number is $k$ in the distribution network.

\section{Solution to the Model}

\subsection{Application of the Improved Adaptive Genetic Algorithm}

The genetic algorithm is an optimization algorithm that simulates the biological evolution process. It has been widely used in research on the planning of power systems [27,28]. Since the basic genetic 
algorithm has many problems, such as slow convergence speed, poor stability, and so on, an improved adaptive genetic algorithm is proposed in this paper to solve the model.

(1) Chromosome encoding. In order to facilitate the analysis, this paper assumes that the DG units are all located at load points. The chromosome in the algorithm uses binary encoding, and the corresponding binary bit string of each load point is composed of two parts: the type and capacity of DG. The specific form is as follows:

$$
\phi=\left\{T_{1} S_{1}, T_{2} S_{2}, \cdots, T_{n_{L}} S_{n_{L}}\right\}
$$

where $\Phi$ is a chromosome; $T_{i} S_{i}$ is the type and capacity of DG at load point $I ; T$ represents the type and $S$ represents the capacity.

(2) Selection operator. Based on the elitist strategy, several optimal individuals in the previous generation will be copied directly to the present generation. It can be proven mathematically that the elitist strategy can make the algorithm converge to the global optimal solution with a probability of one [29].

(3) Crossover operator. Based on the improved adaptive crossover operator, the crossover probability of the algorithm will be adaptively adjusted according to the following formula:

$$
P_{c}= \begin{cases}P_{c 1}-\frac{P_{c 1}-P_{c 2}}{f_{\max }-f_{\text {ave }}}\left(f_{\text {mor }}-f_{\text {ave }}\right) & f_{\text {mor }} \geq f_{\text {ave }} \\ P_{c 1} & f_{\text {mor }}<f_{\text {ave }}\end{cases}
$$

where $P_{c}$ is the adjusted crossover probability; $f_{\max }$ is the maximum fitness in the group; $f_{\text {ave }}$ is the average fitness of a generation; $f_{m o r}$ is the larger fitness in the two individuals to cross, $P_{c 1}=0.9$ and $P_{c 2}=0.4$.

(4) Mutation operator. Based on the improved adaptive mutation operator, the adaptive adjustment formula of the mutation probability is as follows:

$$
P_{e}= \begin{cases}P_{e 1}-\frac{P_{e 1}-P_{e 2}}{f_{\max }-f_{\text {ave }}}\left(f_{\max }-f_{e}\right) & f_{e} \geq f_{\text {ave }} \\ P_{e 1} & f_{e}<f_{\text {ave }}\end{cases}
$$

where $P_{e}$ is the adjusted mutation probability; $f_{e}$ is the fitness of the individual to mutate; $P_{e 1}=0.9$ and $P_{e 2}=0.4$.

\subsection{General Steps}

Based on the method proposed in this paper, the general steps of multi-type DG planning are summarized as follows:

(1) Classification of daily load sequences. The daily load sequences throughout the year at each load point in the distribution network should be divided into $A$ categories according to different seasons and date types; then, the number of days included in each category should be recorded.

(2) Calculation of typical daily load sequence. For each category of the daily load sequences, the typical daily load sequence can be calculated according to Equation (1). If the number of the load points in the distribution network is $n_{L}$, then the total number of the typical daily load sequences obtained after calculation is $A n_{L}$. 
(3) Calculation of a typical daily output sequence of DG per unit capacity. For each category of the daily load sequences, the typical daily output sequence of DG per unit capacity can be calculated according to Equation (2). If the number of the types of DG to be selected is $B$, then the total number of the typical daily output sequences of DG per unit capacity obtained after calculation is $A B n L$.

(4) Calculation of a typical daily output sequence of DG. For each load point in the individual, the typical daily output sequence of DG per unit capacity should be selected first according to the type and capacity of the DG; then, the typical daily output sequence of DG at each load point can be obtained through the conversion of the typical daily output sequence of DG per unit capacity.

(5) Power flow calculation. After the superposition of the typical daily output sequence of DG and the typical daily load sequence, the power flow calculation can be started. If the constraint conditions cannot be met, the fitness of the individual should be set to zero directly; otherwise, the fitness will be calculated according to the fitness function.

(6) Analysis of the results. Repeat Steps 4 and 5 until the preset maximum number of generations is reached; then, the individual whose fitness is the largest is the optimal solution to the model. This indicates the type and capacity of the DG that should be connected to each load point.

The specific calculation process is shown in Figure 6.

Figure 6. Flow chart of the proposed algorithm based on GA.

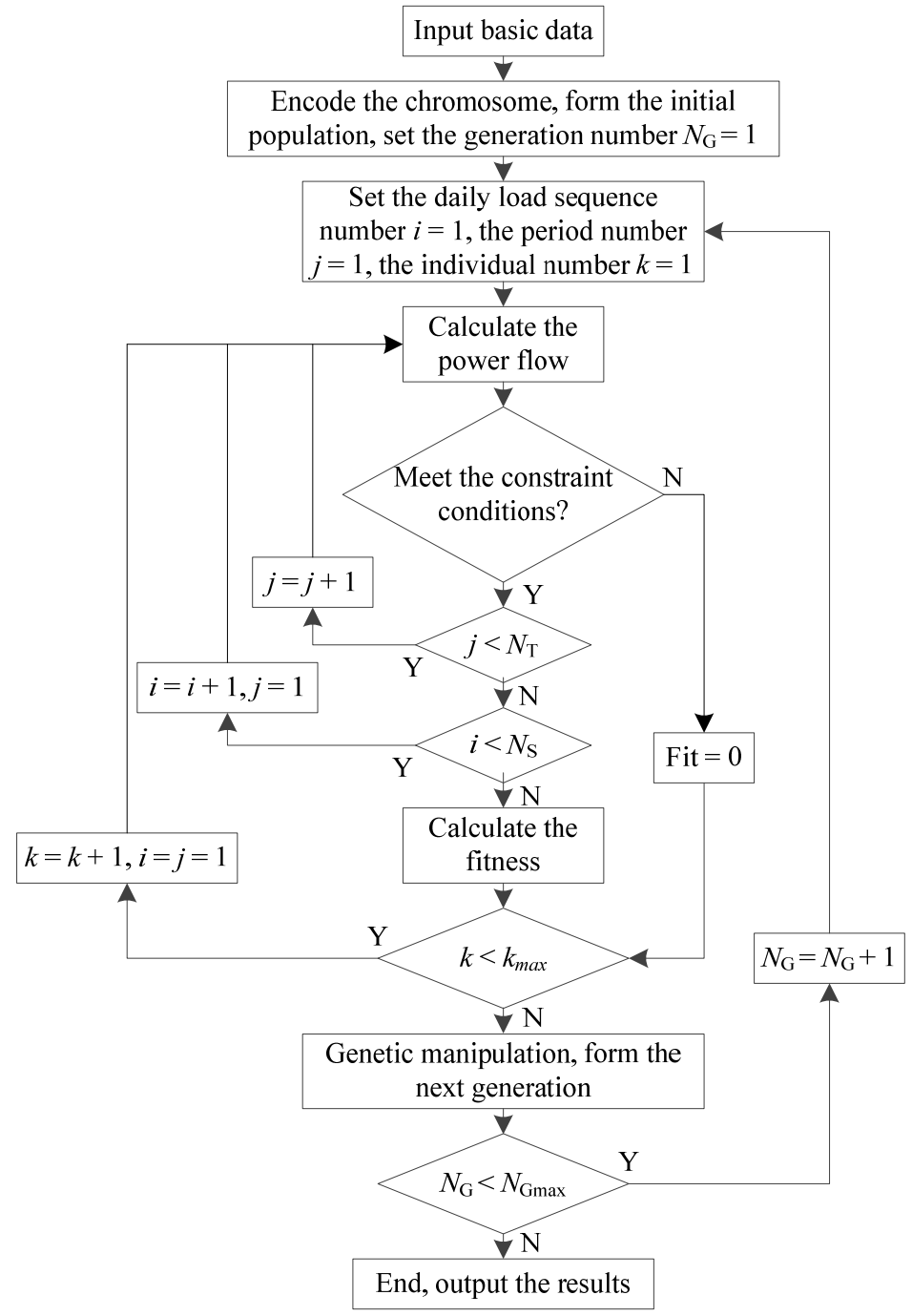




\section{Case Study}

\subsection{Overview of the Test System}

In this paper, the IEEE 14-node distribution system is employed for the planning of multi-type DG. Normally, the distribution network is operated in open-loop; therefore, Branches 14, 15 and 16 have been removed to form a single-supply radial network with 14 nodes and 13 branches. Figure 7 shows the schematic of the system.

Figure 7. Schematic of the IEEE 14-node system.

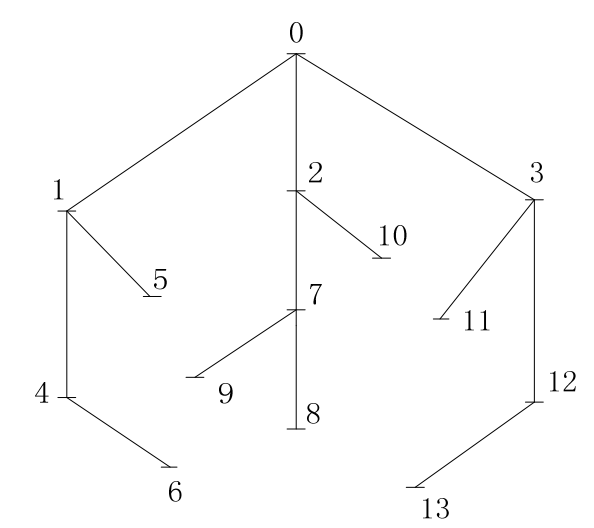

In Figure 7, Node 0 is the slack bus and the remaining nodes are all load connection points. In the system, the base power for calculation is $100 \mathrm{MVA}$ (Mega Vlot-Ampere); the base voltage is $23 \mathrm{kV}$, and the total load is $28.7+7.75 \mathrm{MVA}$. This paper assumes that all of the load points are allowed to connect to DG. The candidate types include wind power generation (WG), photovoltaic power generation (PV), micro gas turbine (MT) and fuel cell (FC). For each candidate, the capacity is $\mathrm{K} \times 100 \mathrm{kVA}$ $(\mathrm{K}=1,2, \ldots, 7)$. Other parameters and values are shown in Table 2.

Table 2. Parameters and values in the example. WG, wind power generation; PV, photovoltaic power generation; MT, micro gas turbine; FC, fuel cell.

\begin{tabular}{cc}
\hline Parameters & Values \\
\hline The installation cost of WG & $7,000 \mathrm{CNY} / \mathrm{kW}$ \\
The installation cost of PV & $10,000 \mathrm{CNY} / \mathrm{kW}$ \\
The installation cost of MT & $3,000 \mathrm{CNY} / \mathrm{kW}$ \\
The installation cost of FC & $12,000 \mathrm{CNY} / \mathrm{kW}$ \\
Annual interest rate & $3 \%$ \\
Load growth rate & $1 \%$ \\
The price of the lost electricity & $0.35 \mathrm{CNY} / \mathrm{kWh}$ \\
The purchase price of the electricity & $0.35 \mathrm{CNY} / \mathrm{kWh}$ \\
The sell price of the electricity & $0.5 \mathrm{CNY} / \mathrm{kWh}$ \\
The subsidy price of the electricity & $1.0 \mathrm{CNY} / \mathrm{kWh}$ \\
The power factor of DG & 0.9 \\
The economic life of DG & 25 years \\
\hline
\end{tabular}


The type of DG is expressed using two-binary code: 00 represents WG, 01 represents PV, 10 represents MT and 11 represents FC. The capacity of DG connected to each load point is expressed using three-binary code. In order to achieve complementary effects and to ensure the stability of the system, this paper defines that the minimum capacity of each type of grid-connected DG is $10 \%$ of the total capacity, which cannot exceed $10 \%$ of the total load of the system.

\subsection{Analysis of Different Optimization Schemes}

Scheme 1: without considering the relationships among different interest groups, set $\lambda_{1}=\lambda_{2}=\lambda_{3}=\lambda_{4}=$ $\lambda_{6}=1$ and $\lambda_{5}=0$; then, the maximum net income brought by grid-connected DG each year can be obtained after the optimization. The optimization scheme is shown in Table 3, and the calculated values of each sub-goal are shown in Table 4.

Table 3. The optimization scheme of DG.

\begin{tabular}{ccc}
\hline Node number & Capacity/kVA & Type \\
\hline 2 & 300 & FC \\
4 & 400 & PV \\
6 & 200 & MT \\
8 & 700 & WG \\
9 & 300 & WG \\
10 & 500 & MT \\
11 & 400 & MT \\
13 & 100 & PV \\
\hline
\end{tabular}

Table 4. The calculated values of each sub-goal.

\begin{tabular}{cc}
\hline Sub-goals & Calculated values/10,000 CNY \\
\hline Loss reduction & 14.72 \\
Delaying the upgrade of lines & 10.44 \\
Environmental protection & 58.80 \\
Saving fuels & 29.20 \\
The fixed investment and maintenance of DG & 109.34 \\
The maximum net income of DG & 3.82 \\
\hline
\end{tabular}

It can be seen that the difference of the capacity between MT and WG is not obvious, which indicates that the economic advantages of MT have been largely reduced by environmental cost and fuel cost. However, PV and FC are difficult for wide use in a short time, due to the higher cost of installation. In addition, as the relationships among different interest groups are not considered, all of the benefits from reducing pollutant emissions and fuel consumption belong to the DG units. These benefits constitute the main economic benefits, making it possible for the DG units to remain profitable. Thus, without considering the relationships among different interest groups, the economic benefits of DG can be represented objectively. 
Scheme 2: considering the interests of the DG owner only, assume that all of the DG units belong to the power supply company, and set $\lambda_{1}=\lambda_{2}=\lambda_{3}=\lambda_{4}=\lambda_{5}=\lambda_{6}=1$ according to the benefits of the power supply company; then, the maximum net income for the power supply company each year can be obtained from the optimization. The locations, capacities and types of the DG units are shown in Table 5, and the calculated values of each sub-goal are shown in Table 6.

Table 5. The optimization result of DG.

\begin{tabular}{ccc}
\hline Node number & Capacity/kVA & Type \\
\hline 1 & 200 & MT \\
3 & 100 & PV \\
4 & 300 & WG \\
6 & 300 & PV \\
8 & 600 & WG \\
9 & 400 & MT \\
10 & 400 & MT \\
11 & 300 & FC \\
12 & 200 & MT \\
\hline
\end{tabular}

Table 6. The calculated values of each sub-goal.

\begin{tabular}{cc}
\hline Sub-goals & Calculated values/10,000 CNY \\
\hline Loss reduction & 13.81 \\
Delaying the upgrade of lines & 9.78 \\
Environmental protection & -6.05 \\
Saving fuels & -103.28 \\
Sale of electricity and subsidies & 616.52 \\
The fixed investment and maintenance of DG & 101.40 \\
The maximum net income for the power supply company & 429.38 \\
\hline
\end{tabular}

Compared to Scheme 1, the total capacity of DG is reduced slightly, but the capacity of MT is increased. This is mainly because the pollution fines of MT are much less than the values of environmental cost. In addition, the power supply company is subject to financial loss if there is no subsidies from the government. This suggests that the economic benefits of DG cannot be well represented if the only factor to be considered is the interests of the owner of DG. This is also one of the reasons that it is difficult to popularize DG.

\subsection{Analysis of the Convergence of the Algorithm}

Figure 8 is the process of solving Scheme 1 with the algorithm in this paper. It reflects the changes of the solution. As can be seen, when the number of iterations reaches about 70 , the calculation results tend to be stable, which shows the good convergence of the proposed algorithm. 
Figure 8. Solution convergence with the number of iterations.

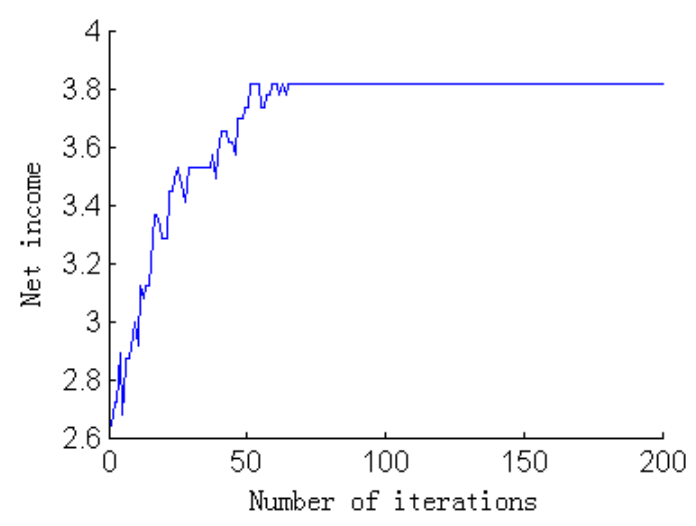

\section{Conclusions}

A method for multi-type DG planning is proposed in this paper to provide a detailed analysis of the income items and investment items brought by grid-connected DG. The approach can measure the economic benefits of DG objectively and also help all parties make decisions on the planning scheme according to their own interests. Calculation results show that the method is feasible and effective at providing important recommendations for the planning and design of DG.

Without considering the relationships among different interest groups, the economic benefits of DG can be represented the most objectively. However, if the only factor to be considered is the interests of the DG owner, most of the economic benefits will be concealed, when the owner can only remain profitable through seeking state subsidies.

Under the current situation, the net income brought by grid-connected DG is not optimistic. However, it is expected that with the reduction of DG costs, the rise in the price of conventional energy resources and possible government low carbon emission subsidy schemes, the economic benefits of DG will grow significantly.

\section{Acknowledgments}

The authors would like to acknowledge the financial support from the National Natural Science Foundation of China (No. 51177047) and the Fundamental Research Funds for the Central Universities (No. 12MS107).

\section{Author Contributions}

The author Jianpeng Liu carried out the main research tasks and wrote the full manuscript, and the author Yajing Gao proposed the original idea, analyzed and double-checked the results and the whole manuscript. Jin Yang contributed to the writing and summarizing proposed ideas, and the authors Haifeng Liang and Jiancheng Zhang provided technical and financial support throughout.

\section{Conflicts of Interest}

The authors declare no conflict of interest. 


\section{References}

1. Parker, P. Integrating Distributed Resources into Electric Utility Distribution Systems; EPRI PEAC Corporation: Palo Alto, CA, USA, 2001.

2. Qian, K.; Yuan, Y.; Shi, X. Environmental benefits analysis of distributed generation. In Proceedings of the 2008 IEEE Power and Energy Society General Meeting - Conversion and Delivery of Electrical Energy in the 21st Century, Pittsburgh, PA, USA, 20-24 July 2008; Volume 29, pp. 11-15.

3. Tran, K.; Vaziri, M. Effects of Dispersed Generation (DG) on distribution systems. In Proceedings of the IEEE Power Engineering Society General Meeting, San Francisco, CA, USA, 12-16 June 2005.

4. Chiradeja, P.; Ramakumar, R. An approach to quantify the technical benefits of distributed generation. IEEE Trans. Energy Convers. 2004, 4, 764-773.

5. Li, B.; Liu, T.; Li, X. Impact of distributed generation on power system voltage stability. Power Syst. Technol. 2009, 3, 84-88.

6. Dong, Y.; Yang, J.; Hu, H. Coordination control of distributed wind-solar hybrid system. Electr. Meas. Instrum. 2012, 554, 48-51.

7. Song, X.; Ye, L. Optimization configuration of wind/solar/diesel hybrid power generation system. Power Syst. Clean Energy 2011, 5, 66-72.

8. Fu, L.; Wang, S.; Zhang, Y.; Zhang, C.; Dong, P. Optimal selection and configuration of multi-types of distributed generators in distribution network. Power Syst. Technol. 2012, 1, 79-84.

9. Yang, Q.; Ma, S.; Song, Y. Comprehensive evaluation of distributed generation planning scheme. Power Syst. Technol. 2012, 2, 212-217.

10. Zhang, Q.; Wang, G.; Zhai, H. Index system for distributed generation development and comprehensive benefits in China. East China Electr. Power 2012, 7, 1248-1251.

11. Hu, H.; Wu, S.; Xia, X. Computing the maximum penetration level of multiple distributed generators in distribution network taking into account voltage regulation constraints. Proc. CSEE 2006, 19, 13-19.

12. Lu, H.; Zhou, H. Environmental cost analysis of power plants. Environ. Prot. 2004, 4, 51-54.

13. Celli, G.; Ghiani, E.; Moccis, S. A multiobjective evolutionary algorithm for the sizing and sitting of distributed generation. IEEE Trans. Power Syst. 2005, 2, 750-757.

14. Li, L.; Tang, W.; Bai, M.; Zhang, L.; Lv, T. Multi-objective locating and sizing of distributed generators based on time-sequence characteristics. Autom. Electr. Power Syst. 2013, 3, 58-63.

15. Zheng, Z.; Ai, Q.; Gu, C. Multiobjective allocation of distributed generation considering environmental factor. Proc. CSEE 2009, 13, 23-28.

16. Li, R.; Shen, X.; Zhong, C.; Yang, T. Multi-objective planning of distributed generation considering environmental benefit. Power Syst. Technol. 2014, 6, 1471-1478.

17. Cui, H.; Dai, W. Multi-objective optimal allocation of distributed generation in smart grid. In Proceedings of the 2011 International Conference on Electrical and Control Engineering (ICECE), Yichang, China, 16-18 September 2011; pp. 713-717.

18. Zidan, A.; Shaaban, M.F.; El-Saadany, E.F. Long-term multi-objective distribution network planning by DG allocation and feeders' reconfiguration. Electr. Power Syst. Res. 2013, 105, 95-104. 
19. Xu, X.; Chen, K.; Long, Y.; Cheng, H.; Ni, W.; Wang, Z.; Wang, X. Optimal site selection and capacity determination of multi-types of distributed generation in microgrid considering environment cost and timing characteristics. Power Syst. Technol. 2013, 4, 914-921.

20. Liao, N.; Hu, Z.; Ma, Y.; Lu, W. Review of the short-term load forecasting methods of electric power system. Power Syst. Prot. Control 2011, 1, 147-152.

21. Sun, Q.; Yao, J.; Zhao, J.; Jin, M.; Mao, L.; Mao, T. Short-term bus load integrated forecasting based on selecting optimal intersection similar days. Proc. CSEE 2013, 4, 126-134.

22. Lin, H.; Liu, J.; Hao, Z.; Zhu, F.; Wu, G. Short-term load forecasting for holidays based on the similar days'load modification. Power Syst. Prot. Control 2010, 7, 47-51.

23. Suo, L.; Ding, Q.; Han, X. Short-term load forecasting for holidays using fuzzy inference method. Electr. Power 2009, 11, 66-69.

24. Tang, L. The class of DG and its influence of distribute network. Master's Thesis, Hefei University of Technology: Hefei, China, 2007.

25. Zhang, S.; Zhou, S.; Guo, Y.; Wang, X. Seasonal variation of ground wind velocity in recent 47 years in Hebei and its influence factors. J. Arid Land Resour. Environ. 2010, 12, 93-99.

26. Sui, $X$. The distribution network reliability and economy study considering the distribution generation. Master's Thesis, Southwest Jiaotong University: Chengdu, China, 2010.

27. Zhang, L.; Shu, J.; Lin, X. Reactive power planning based on genetic algorithm. Proc. CSEE 2000, 6, 5-8.

28. Zhang, L.; Fan, M. A new model and methodology for distribution network integration planning. Proc. CSEE 2004, 6, 59-64.

29. Ding, M.; Wu, Y. Expansion planning of wind-diesel energy system based on genetic algorithm. Proc. CSEE 2006, 8, 23-27.

(C) 2014 by the authors; licensee MDPI, Basel, Switzerland. This article is an open access article distributed under the terms and conditions of the Creative Commons Attribution license (http://creativecommons.org/licenses/by/4.0/). 